\title{
Risk Assessment of Hazards Due to the Installation and Maintenance of Onshore Wind Turbines
}

\author{
Albara M. Mustafa \\ Institute of Building, Energy and Material Technology \\ Uit the Arctic University of Norway \\ Narvik, Norway \\ albara.m.mustafa@uit.no
}

\author{
Aziz Al-Mahadin \\ Aviation Engineering Department, DBM \\ Higher Colleges of Technology \\ Dubai, UAE \\ aalmahadin@hct.ac.ae
}

\begin{abstract}
In this work, an assessment of four types of risks is carried out for wind turbines during four phases, namely: transportation, installation, operation and maintenance. This work mainly focuses on onshore type of wind turbines and briefly mentioning the offshore wind turbines. The introduction gives an overview of the main parts and components of wind turbine, in addition to discussing the process of risk assessment and the procedure to be followed in this study. The paper focuses on the following four risks: the risk of transporting large-scale wind turbine parts and components, the risk of workers slipping, tripping and falling during installation and maintenance of wind turbines, the risk of working in confined spaces, and finally the risk of ice accretion and irregular shedding when the wind turbine is in operation phase or even when it is stationary. The last type of risk is highly observed in cold climate regions. The four mentioned types of risks are the main ones out of the many risks that could appear during transporting, installing, operating and maintaining wind turbines. The main aim of this work is to contribute in the proper risk assessment of potential hazards, which enhances the ability to devise passive and active protection measures to reduce the effects of a catastrophic event.
\end{abstract}

Keywords-risk assessment; hazard identification; risk analysis; risk evaluation; wind turbine

\section{INTRODUCTION}

There is a rising commitment towards utilizing clean energy across the world. Wind turbines happen to be an efficient choice to meet some of the demand for electricity. A group of wind turbines connected to each other by electrical cables forms a wind farm. A wind farm produces 17 to 39 times the power it consumes, which is more efficient when compared to other forms of energy such as nuclear power (16 times) or coal (11 times) [1].

An onshore wind turbine sits on a structural support usually made of concrete, called the Foundation, the importance of a foundation is to transfer the horizontal and vertical forces acting on the wind turbine to the surrounding ground. Whether the case is offshore or onshore, a wind turbine would essentially consist of the same parts. The first component of a wind turbine is the tower whose height varies depending on the site and the available wind speed. Going from bottom to top, the cross section of the tower becomes narrower. Normally, a ladder is mounted inside of the wind turbine's tower. However, modern towers have lifts [2]. On the top of the tower, lies another part called the nacelle. The nacelle holds the needed equipment to convert wind energy into electricity, such as gearbox, braking system, generator and yawing mechanism to correct the direction of the nacelle to face the wind direction. The wind turbine blades are connected to the nacelle through a hub. Generated electricity is transferred via cables to a step-up transformer located at the base of the wind turbine [3].

Wind-turbines are constructions that are susceptible to windy conditions and they have many mechanical and electrical moving parts and instruments. Therefore, there is a great need for awareness of the types of hazards that workers might face when dealing with wind turbines. This research aims towards investigating the hazards that might take place during the installation and maintenance phases of mainly onshore wind turbines by utilizing risk assessment methodologies to identify the probable hazards, their level of risk and ways of mitigation and control.

\section{RISK ASSESSMENT}

According to the Canadian Standards Association (CSA) Z1002 Standard "Occupational health and safety - Hazard identification and elimination and risk assessment and control", the following terms used in the risk assessment procedure are defined as follows [4], [5]:

- Risk is identified as the combination of the likelihood of the occurrence of a harm and the severity of that harm.

- Risk assessment is the overall process of hazard identification, risk analysis, and risk evaluation.

- Risk assessment provides an understanding of risks, their causes, consequences, and their probabilities.

- Hazard identification is the process of finding, listing and characterizing hazards.

- Risk analysis is a process for comprehending the nature of hazards and determining the level of risk.

- Risk evaluation is the process of comparing an estimated risk against given risk criteria to determine the significance of the risk.

A decision is taken based on risk evaluation in order to mitigate or control the risk. This step is known as Risk Control. 
Before implementing risk control measures, the level of risk has to be determined in order to decide which hazard to start treating [5]. One of the conventional methods used to rank or prioritize risks is the Risk Matrix shown in Table I, where the vertical column shows the likelihood of the risk to take place and the horizontal row shows risk severity if it happens. The combination between likelihood and severity in this qualitative method determines the level of the risk; consequently a decision can be made on which measures should be taken in order to control and mitigate the risk.

The following sections discuss various types of risks during wind turbines transportation, installation, operation and maintenance.

Table I. Risk matrix showing the risk likelihood and severity [6].

\begin{tabular}{|c|c|c|c|c|c|}
\hline \multicolumn{2}{|c|}{ A } & B & C & D & E \\
\hline E & Low Med & Medium & Med Hi & High & High \\
\hline D & Low & Low Med & Medium & Med Hi & High \\
\hline C & Low & Low Med & Medium & Med Hi & Med Hi \\
\hline B & Low & Low Med & Low Med & Medium & Med Hi \\
\hline A & Low & Low & Low Med & Medium & Medium \\
\hline
\end{tabular}

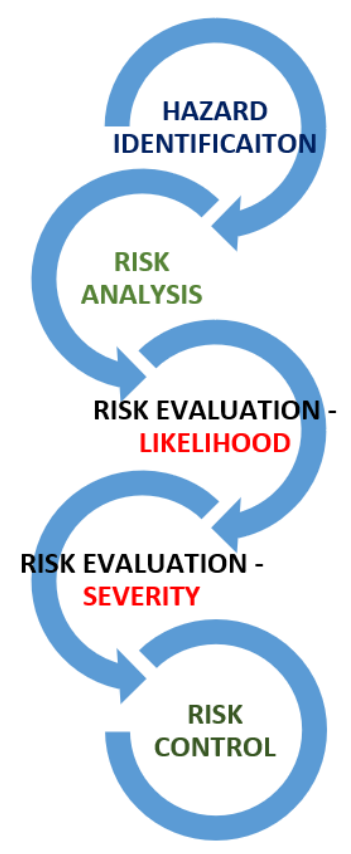

Fig. 1. Risk assessment process [7].

\section{RISK OF TRANSPORTING LARGE-SCALE WIND TURBINE COMPONENTS}

\section{A. Hazard Identification.}

Wind turbines differ in sizes and capacities. Generally, larger wind turbines are able to deliver more electricity at lower cost. This is because some costs related to building a wind turbine are independent of its size, such as the cost of the foundation, building new roads and electrical grid connections [7]. Transporting large components on roads can cause injury to people and damage to materials. The likelihood and severity of hazard may increase while transporting large wind turbine components in particular at narrow roads with heavy traffic, or difficult road terrains.

\section{B. Risk Analysis}

Transporting large wind turbine components faces many challenges. As for onshore wind turbines, components might have to be transported via existing public roads, which allows for limited movement and poses high risk to vehicles on the road when conveying such parts to the construction site. On the other hand, marine transportation and installation equipment allow better handling of larger parts, therefor, offshore wind turbines tend to be larger than onshore ones [2].

As an example of large wind turbines parts, Siemens is working with other partners to create $6 \mathrm{MW}$ offshore wind turbines, the size of one blade of these turbines is a fraction smaller than An A380's wingspan. Siemens says that one 6MW wind turbine is enough to supply about 6,000 European households with electricity. Fig. 2, shows a special vehicle transporting a large-scale wind turbine blade at speed of 60 Kilometer per hour over a distance of 575 kilometers to the construction site [8]. Another example is the widely used GE 1.5-megawatt wind turbine, which consists of almost $116 \mathrm{ft}$. blade mounted on top of $212 \mathrm{ft}$. tower. The blade assembly of that wind turbine weighs more than 36 tons and the tower weighs about 71 tons [9].

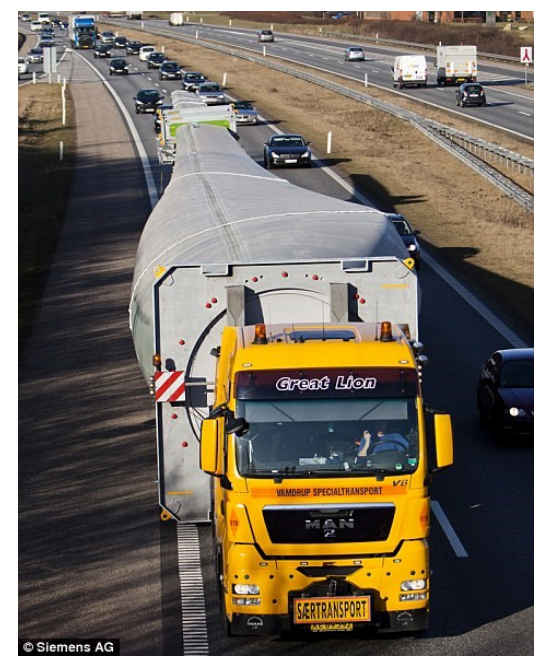

Fig. 2. Special vehicle transporting large wind turbine blades [7] 


\section{Risk Evaluation / Likelihood.}

The probability that an accident would occur when transporting large components of wind turbine depends on many factors, such as the number of vehicles on the road, the condition of the road, whether the road has many twists and curves and the speed limit of the road. Furthermore, accident likelihood can increase depending on many other factors including: driver capability, weather, transported components size, shape and loading method. Referring to the risk matrix shown in Fig.1, there is a possibility that an accident would occur when transporting large wind turbine parts. However, the degree of probability dependents on the above various factors.

\section{Risk Evaluation / Severity.}

Accidents resulting from transporting large wind turbines parts could cause serious damages to roads, other vehicles and to passengers and pedestrians. The probability of accidents taking place in similar situation depends on multiple factors.

If transportation is done via public roads, then accidents happening in this case are highly severe. Therefor drivers should be highly cautious when driving nearby vehicles transporting such parts. Professionals who are responsible for such transportation should take all the necessary measures to reduce the likelihood and severity of accidents such as selecting transportation time, using the proper vehicles and skilled drivers, and cooperation with traffic police, etc.

\section{E. Risk Control.}

In some cases, new roads are built so that wind turbines are transported and to be installed in rural areas. However, in many other cases, there is still a need to use public roads to transport large wind turbine components. Therefore, to control this kind of risk, some measures should be taken, such as avoiding rush hours and highly dense roads, avoid exceeding speed limit determined for this kind of transportation. Other measures include changing the layout of the road, such as widening narrow roads and straightening curved roads. Fig. 3, shows analysis of this risk.

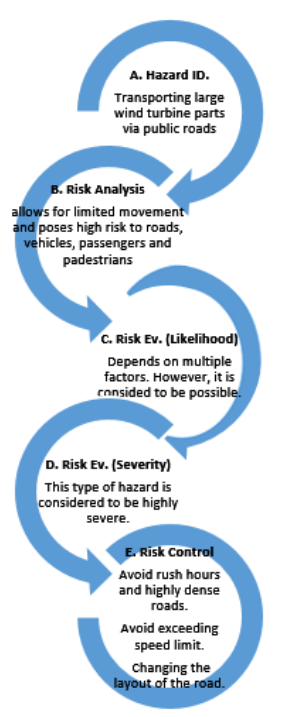

Fig. 3. Risk analysis of transporting large-scale wind turbine parts.

\section{RISK OF WORKERS SLIPPING, TRIPPING AND FALLING}

\section{A. Hazard Identification.}

Certain parts in a wind turbine will be in motion for long periods and others are exposed to harsh weather conditions. To make sure the wind turbine stays functional; these parts have to be well maintained. Regular maintenance to wind turbines includes maintaining air filters, brake pads, tower bolts and lubrication [1]. Most of the required maintenance is done to main components located in the nacelle, on top of the tower, such as the rotor, gearbox, yawing mechanism etc. Sending maintenance crews to do the required maintenance to these parts may cause certain types of hazards, such as slipping, tripping and falling.

\section{B. Risk Analysis.}

Climbing a wind turbine requires high stamina. As mentioned earlier the GE 1.5-megawatt wind turbine tower is almost $212 \mathrm{ft}$. in height [9], which means that workers have to climb high altitudes using ladders mounted inside the wind turbine tower. However, in some cases, modern wind turbines may have electrical lifts which can reduce certain types of hazard [2]. Maintenance crews are subjected to the risk of slipping and tripping when climbing wind turbine towers. The risk becomes more prominent as they climb higher, since the cross-sectional area of the wind turbine's tower becomes slightly narrower with height. However, ladders must have landing platforms every 30 foot in case they were equipped with cage or well. However, if they were not equipped with cages or wells; the landing platform must be every 20 foot as per regulations of Occupational Safety and Health Administration (OSHA) [10].

Moreover, workers are subjected to falling hazards during installation and maintenance of wind turbines. They may need to access different wind turbine sections at elevations above 100 foot for welding, fitting, maintaining and installing test equipment and electrical cables. Therefore, safety measures become mandatory in order to avoid the risk of falling off high altitudes. This means ensuring equipment are suitable, stable, strong, and maintained regularly. Workers should also receive the required training and work only when their health and psychological conditions are appropriate.

\section{Risk Evaluation / Likelihood}

The likelihood of slipping, tripping and falling should not be so high if the proper safety measures and regulations are diligently considered. This includes utilization of the correct resources, workers are trained, fit, and put on their Personal Protective Equipment (PPE).

\section{Risk Evaluation / Severity.}

Severity of slipping and tripping while climbing up wind turbine's ladders should not be high. However, the risk of falling off the wind turbine while carrying out the required maintenance or installing parts is high. But the actual degree of severity depends on the particular case and should be decided after studying all prevailing conditions and influencing factors. 


\section{E. Risk Control.}

When it comes to wind turbine maintenance, it is not feasible to send maintenance crews to check manually on each wind turbine, the problem becomes more prominent in the case of wind farms. Therefore, utilizing technological instruments for remote monitoring to provide information regarding the functionality of the wind turbine is a feasible and an efficient option. It reduces risk and costs of sending workers for manual check of the wind turbines. As per OSHA standards, maintenance crews working in such environment and exposed to fall hazards of 4 foot and more must be protected by railing. If such railing is not available, workers must wear the proper PPE such as a personal fall arrest system or a safety net. Fig. 4, shows risk analysis of workers slipping, tripping and falling.

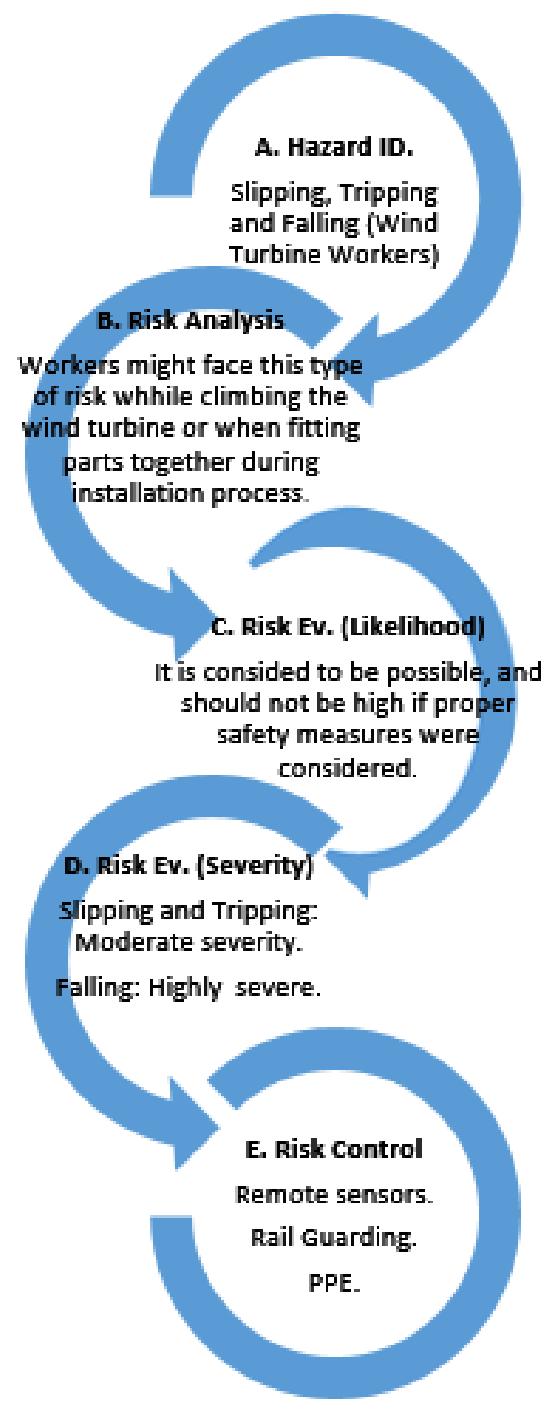

Fig. 4. Risk analysis of workers slipping, tripping and falling.

\section{RISK OF WORKING IN CONFINED SPACES}

\section{A. Hazard Identification.}

As per the OSHA, for a place of work to be defined as confined space, it should have the following characteristics [11]:

- The worker can enter the space fully and perform required work.

- The space is not designed to be permanently occupied by the worker.

- Exit and entry out of and into the confined space are limited.

As mentioned earlier in the hazard identification part for the risk of workers slipping, tripping and falling, most wind turbine maintenance work takes place for components located in the nacelle, which lies on top of the wind turbine's tower. All nacelles configurations are classified as confined spaces for the purposes of this study [11]. Within confined spaces number of hazards may exist including: lack of oxygen, presence of gases and fumes, hot conditions, etc.

\section{B. Risk Analysis.}

Wind turbine maintenance crews might encounter different types of risks during their maintenance work in confined spaces, mainly in the nacelle. Encountered risks might be due to different reasons such as low oxygen environment, resulting from poor ventilation, which can lead to asphyxiation, or accumulated hazardous gases resulting from wind turbine catching on fire. It is stated that wind turbines are natural targets for lightning strikes [12], the rising current from lightning strikes could lead to harm personnel, catastrophic equipment damage, and even to fire [13]. Fire can be discussed as a separate hazard; however, it is mentioned in this paper as a related hazard to confined spaces in wind turbines. In addition to lightning strikes, fire hazards can result from electrical parts, insulations made of combustible materials and from lubricants used for lubricating mechanical parts in the nacelle [14].

\section{Risk Evaluation / Likelihood}

There is a high probability that workers would face confined space hazards when carrying out the needed maintenance inside the nacelle. The hazards are caused mainly by inadequate ventilation leading to low oxygen environment, or toxic gases and fumes due to lubricants and fire caused by different reasons as mentioned earlier.

\section{Risk Evaluation / Severity.}

Confined space hazards are highly severe. Such hazards can lead to asphyxiation, toxicity and eventually to death if proper safety precautions are not considered. In addition to toxic gases resulting from fire hazards, fire can cause highly severe burns to workers while working inside the nacelle, which is recognized as a confined space. 


\section{E. Risk Control.}

In order to control confined space hazards, different measures have to be considered depending on the type of hazard in the confined space. However, it is the duty of the employer to develop a written procedure that details the steps that personnel should take to ensure working safely inside the confined space. Employees should also be trained and have permit to enter confined spaces. As for low oxygen environment or the presence of toxic or flammable gases in the nacelle, workers can perform air sampling before entering the nacelle. Gas Monitor Device is an important tool that should be available with workers when entering confined spaces [11].

To mitigate fire hazards resulting from lightning strikes, a good grounding system is mandatory to protect electronic equipment, blades and the nacelle from serious damages. Spotting lightning strikes before getting to the wind farm is a preventive way of protecting workers, which can be done via utilizing advanced weather hazard real time warning systems [12]. Moreover, workers must be trained to use portable fire extinguishers to deal with fire hazards. A fire extinguishing system must be installed inside the nacelle. In addition, fire detection and emergency alarm systems should be installed inside the nacelle to provide early warning to workers to escape [14]. Fig. 5, shows risk analysis of working in confined spaces.

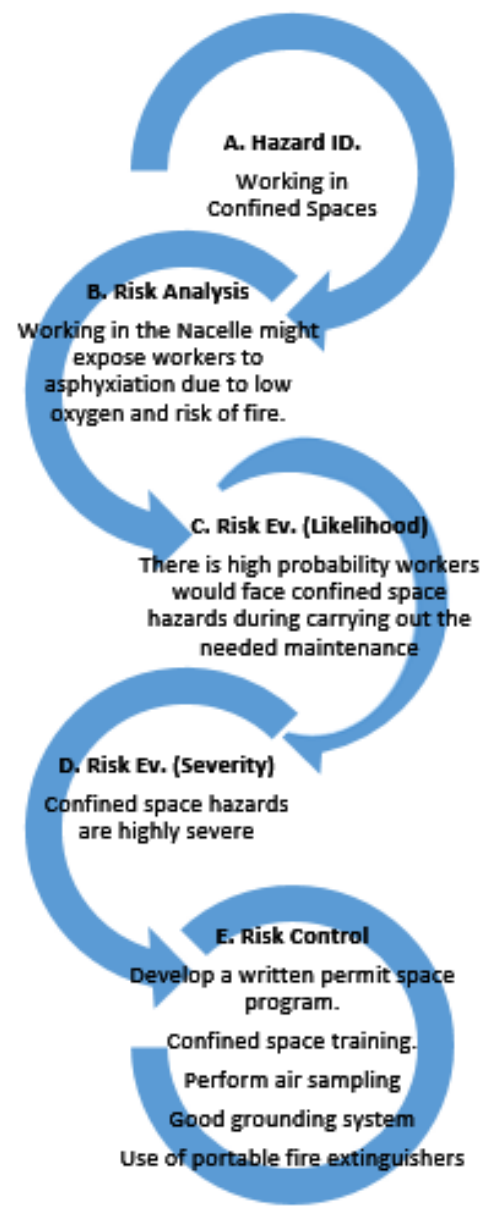

Fig. 5. Risk analysis of working in confined spaces.

\section{RISK OF ICE ACCRETION AND IRREGULAR SHEDDING ON WIND TURBINES}

\section{A. Hazard Identification.}

Most of wind energy investments take place in the northern part of Europe and the Arctic region, where the cold climate affects the operability of wind turbines and poses serious challenges to the wind energy industry through ice accretion on wind turbine blades. The accreted ice is not uniformly distributed over the blades of a wind turbine; it is observed that more ice is accreted over the tip of the blades compared to the root sections [15], [16]. Annual power loss due to ice accretion ranges between $20 \%$ and $50 \%$ [17]. Ice fragments shed from wind turbines may cause injury to people and damage to surroundings including neighboring turbines.

\section{B. Risk Analysis.}

The load of accrete ice can reach up to $50 \%$ of the weight of a blade structural weight [18], which poses serious risk to the structural integrity of the wind turbine and to the surroundings. Ice accretion and irregular shedding on blades can lead to load imbalance, increased fatigue loads, wind turbine vibrations [19]. Moreover, there is a possible danger that accumulated ice is thrown away while melting from the blades during rotating due to centrifugal force.

\section{Risk Evaluation / Likelihood.}

The likelihood of ice accretion and irregular shedding on wind turbine blades is very likely, especially in the arctic region due to the harsh weather in that part of the world. However, the exact likelihood depends on number of factors including the use of de-icing technologies.

\section{Risk Evaluation / Severity.}

Ice accretion on wind turbine blades imposes serious danger to the surroundings in case ice falls off the blades of the wind turbine. Ice shedding may cause injuries to humans and animals. Housing complexes, agricultural infrastructure and industrial plants are also prone to danger from ice shedding if they were built within the range of ice falling from wind turbine blades.

\section{E. Risk Control.}

Safety measures should include constructing of wind turbines in uninhabited locations, and away from roads, agricultural infrastructure and industrial plants. Moreover, wind turbines must be built with safe distance between them to avoid causing damage to each other. A distance of about 250 meters from the wind turbine should be left to minimize the risk of being struck by falling ice during operation [20] and 50 meters when the blades are stationary [21]. Electrical heating of blades can help in preventing or reducing ice accretion and shedding. However, this means certain amount of power is lost to overcome the icing problem. It is stated that a thermal antiicing system requires an amount of power equal to at least $25 \%$ of the wind turbine maximum rated power to overcome the ice 
accretion [22], [23]. Fig. 6, shows risk analysis of ice accretion and irregular shedding on wind turbines.

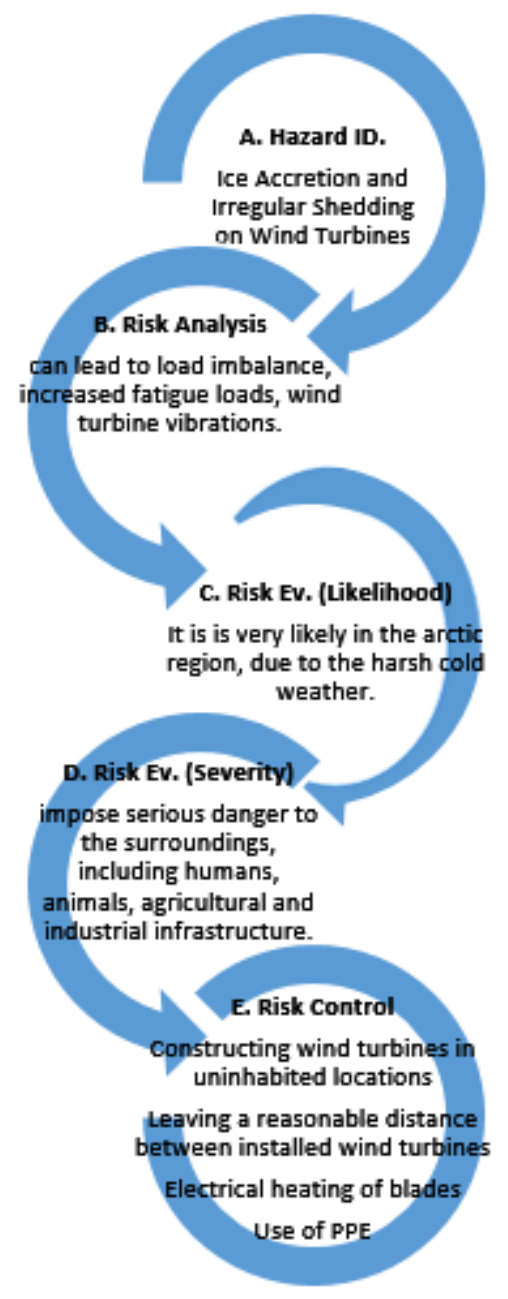

Fig. 6. Risk analysis of ice accretion and irregular shedding on wind turbines.

\section{SUMMARY AND RECOMMENDATIONS}

This paper has presented an assessment of different types of risks that may be happening due to wind turbines utilization. The investigation introduces a systematic methodology for risk assessment of potential hazards in order to establish the required measures needed for risk prevention and mitigation.

The range of risks related to transportation, installation, operation and maintenance of wind turbines is wide. This paper focused on four types of risks affecting surrounding environment and wind turbine workers. The risk of transporting huge parts and components of wind turbines is high and may affect people passing by these parts while being transported. Wind turbine workers are susceptible to many types of hazards, when installing and maintaining wind turbines, like slipping, tripping and falling. The impact of confined spaces and fire hazards on maintenance workers was also discussed. Lastly, ice accretion and irregular shedding on wind turbines is a hazard that may affect people, the surrounding area, and the neighboring wind turbines. This hazard is clearly noticed in the arctic region and the similar world-wide areas.

There are different measures that can be taken to control and mitigate hazards. These measures include: the use of proper technologies, adhering to regulations, using proper equipment, ensuring the correct training, and most importantly performing risk assessment per the particular case.

There are many other types of hazards that has not been discussed in this work, among them electrical hazards, falling objects, and noise hazards which can be areas for future investigations. The following actions are recommended:

- Investigating other types of hazards that may happen due to utilization of wind turbines.

- Carrying out risk assessment for any process that may present hazard to people or damage to equipment.

- Utilizing the presented method in order to reduce the risk that may accompany the use of wind turbines and the similar systems.

- Utilizing other risk assessment tools to identify hazards related to wind turbines, such as What If Analysis, Hazard and Operability (HAZOP), Failure Mode and Effect Analysis (FMEA), Fault Tree Analysis (FTA) and Event Tree Analysis (ETA).

\section{CONCLUSION}

Wind turbine transport, installation, maintenance and operation may cause different types of hazards which should be analyzed and managed in order to reduce the likelihood and severity of incidents and accidents. There are different kinds of measures that can be taken which help to contain hazards within acceptable limits. The selection of proper measures are dependent on the particular case and the prevailing conditions. The systematic process of risk assessment provides clear understanding of the potential hazards and their causes. It makes it easier to identify possible hazards, analyze and evaluate the risks, and eventually lay out the proper measures to control these risks.

\section{ACKNOWLEDGMENT}

The authors convey their gratitude to the support and encouragements received from Dubai Men's College, the Higher Colleges of Technology, UAE, and the Institute of Building, Energy and Material Technology, Uit the Arctic University of Norway, Norway in conducting this research

\section{REFERENCES}

[1] G. Davis, "Wind Turbine (Windmill) Safety and Maintenance," Safety Services, 3rd September 2013.

[2] N. A. O. Sciences, Worker Health and Safety on Offshore Wind Farms Special Report 310, vol. Chapter 2, Washington, D.C., 2013.

[3] Patreon, Director, How do Wind Turbines work? Learn Engineering, Jul 28, 2015.

[4] ISO/IEC Guide, 51:2014, "Safety aspects - Guidelines for their inclusion in standards", Third edition, 2014-04-01. 
[5] M. K. D. Valis, "Selected Overview Of Risk Assessment Techniques", Problemy Eksploatacji, vol. 4, pp. 19-32, 2009.

[6] V. Dumbravă. "Using Probability - Impact Matrix in Analysis and Risk Assessment Projects", Journal of Knowledge Management, Economics and Information Technology, 76 Special Issue, December 2013.

[7] A. M. Mustafa, W. Solvang, E. Dykes and M. Y. Mustafa, "Assessment of wind induced hazards on winterised offshore structures using the cognitive approach," in 5th IEEE Conference on Cognitive Infocommunications (CogInfoCom), Napoli, Italy, 2014.

[8] J. Nader and P. Fuglsang, "Characteristics of The Wind Turbine Blades Industry" Siemens AG 2016.

[9] S. Engström, T. Lyrner, M. Hassanzadeh, T. Stalin and J. Johansson. "Tall towers for large wind turbines", Report from Vindforsk project V342, July 2010.

[10] Occupational Safety and Health Administration (OSHA), "Stairways and Ladders: A Guide to OSHA Rules", 3124-12R, 2003.

[11] Occupational Safety and Health Administration (OSHA), "Confined Spaces", confined-space standard, 29 CFR 1910.146.

[12] Occupational Safety and Health Administration (OSHA), "Green Job Hazards: Wind Energy - Confined Spaces", 2017.

[13] K. Brashear, "Lightning and Surge Protection Of Modern Electronic Systems" ILD Technologies, LLC San Antonio, TX.

[14] R. Okeke, and M. Ehikhamenle, "Design and simulation of gas and fire detector and alarm system with water sprinkle", International Journal of Engineering Research and General Science Volume 5, Issue 1, JanuaryFebruary, 2017 ISSN 2091-2730.
[15] Occupational Safety and Health Administration (OSHA), "Green Job Hazards: Wind Energy - Fires", 2017.

[16] A. S. Alsabagh, "Effect Of Atmospheric Ice Accretion On The Dynamic Performance Of Wind Turbine Blades", Hatfield, UK: University of Hertfordshire, May 2016.

[17] P. \&. F. M. FU, "A CFD Approach for Modeling the Rime-Ice Accretion Process on a Horizontal-Axis Wind Turbine.," J. Wind Eng. Ind. Aerodyn., vol. 98, p. 181-188, 2010.

[18] H. \&. T. B. SEIFERT, "Icing of wind turbines-Final Report.," EnergiesInstitute, D. W. (ed.)., Wilhelmshaven, Finland: Finnish Meteorological Institute, 1996.

[19] ISO, "Atmospheric Icing of Structures," International Standardisation Organisation, I. (ed.)., 2001.

[20] X. B. E. L. \&. N. G. F. Wang, "Experimental Investigation of Energy Losses due to Icing of a Wind Turbine.," in International Conference on Power Engineering, Hangzhou, China, 2007.

[21] C. B. E. \&. S. H. Morgan, "Assessment of Safety Risks Arising from Wind Turbine Icing," BOREAS IV, Hetta, Finland, 1998.

[22] A. \&. L. G. Gadawski, "The Real Truth About Wind Energy, A Literature Review on Wind Turbines in Ontario," INTERNS, S. C. C. (ed.), Ottawa, Canada, 2011.

[23] W. J. N. S. C. S. M. S. \&. B. M. B. Jasinski, "Wind turbine performance under icing conditions," AIAA, ed. AIAA Meeting Papers on Disc, Reno, NV, USA, 1997. 